\title{
Multi-Hypothesis Map-Matching on 3D Navigable Maps using Raw GPS Measurements
}

\author{
Clément Fouque ${ }^{1,2}$, \& Philippe Bonnifait ${ }^{1,2}$ \\ ${ }^{1}$ Université de Technologie de Compiègne (UTC), ${ }^{2}$ CNRS Heudiasyc UMR 6599, France
}

\begin{abstract}
For many road transport applications, maps of the environment where the vehicles evolve are available. This information can contribute to the positioning process itself. In this paper, global positioning on navigable maps is formalized in a general Bayesian framework. Using a tight integration of map data, a generic solution to multi-hypothesis map-matching is described. This method is then applied to the use of raw GPS measurements: pseudoranges and Dopplers. State space equations are given and a marginalized particle filter is proposed to solve efficiently the problem. Experimental results are presented and show that this approach can provide good results even if few satellites are visible.
\end{abstract}

Index Terms-Map-matching, tightly coupled GPS, Bayesian filtering

\section{INTRODUCTION}

During the last decade, many Intelligent Transportation Systems (ITS) applications involving geographical data have been proposed to enhance driving assistance and safety. Such kind of information is commonly stored in a geo-referenced navigable map that describes the road network. To retrieve attributes, the relative location with respect to the map has to be known.

This problem, called map-matching, has been deeply studied in the ITS field of research. Various approaches using either geometrical or topological description of the network have been proposed [1], [2]. Somehow, most of them rely on global position fixes, see [3], [4] for instance. Therefore, their efficiency depends on the availability and the quality of the positioning. To settle these limitations, another approach consists in solving map-matching simultaneously with positioning [5], [6], [7]. Such a tightly-coupled approach increases positioning availability as the number of measurements can be reduced. Precision can also be improved as shown in the paper [8] where GPS differential corrections are elaborated using the map geometry.

In map-matching, the correct road link identification is the main issue, especially in ambiguous situations. When using snapshot or mono-hypothesis approaches like in [3], [5], [6], ambiguity can occur in case of parallel roads or at junctions. Therefore, approaches considering the use of the network topology have been proposed. The authors of [9] have proposed a Multi-Hypothesis Tracking (MHT) approach that exploits the road connectivity. Hypotheses are attached to roads and new hypotheses are created at each new road junction. The authors of [7] choose a constrained Interacting Multiple Model (IMM) approach to handle ambiguities at road junctions. When entering in a road junction area, a bank of map-constrained Extended Kalman Filters (EKF) are used.
Another approach relies on a constrained particle filter (PF) [10]. Here, the evolutions of the particles are constrained by the road geometry and when a junction is reached, particles are allocated to connected roads thanks to the knowledge of the topology of the network.

This approach is here extended to a generic description of Multi-Hypothesis Map-Matching (MHMM). MHMM is a particular positioning problem in which all candidate solutions are searched on the space defined by the map. The problem consists in finding the roads and the locations on the roads simultaneously. In a Bayesian framework, this problem can be stated as a hybrid state estimation problem in the form of a Jump Markov System. According to this description, we investigate the problem of multi-hypothesis tracking in the measurement domain. Here, positioning hypotheses are estimated through a map-constrained marginalized PF that exploits raw GPS measurements, i.e. pseudoranges (PR) and shifts of satellite frequency (called Dopplers in the following), to accomplish Bayesian inference. Therefore, this approach allows estimating the MHMM in a single step without any global position.

The paper is organized as follows. First, the MHMM problem is stated in a generic Bayesian framework and the characterization of positioning hypotheses is described. The following section presents an application of this general method to a specific problem: we consider the use of raw GPS measurements combined with a 3D road map to estimate the vehicle location. For an efficient implementation, a marginalized PF is used that combines both network geometry and topology. Finally, experimental results using few visible satellites are reported to evaluate the solver efficiency regarding to positioning quality and road identification.

\section{BAYESIAN MHMM}

A navigable road map describes here the road network using a geo-referenced carriageway per driving direction. The connections between carriageways are known. In this section, the MHMM is formalized whatever the sensor used and whatever the representation of roads (polylines or clothoids for instance).

\section{A. State-Space model}

A map-based positioning problem can be seen as an hybrid state estimation problem. Each carriageway is identified by a unique identification (ID), denoted $\mathcal{I}$, that is discrete. This description may be extended to address the lane-matching problem [4]. The location on a carriageway is described 
by the curvilinear abscissa, denoted $l$, which is continuous. Some additional parameters may be needed to solve the state observation problem as we will see later on. So, let denote $x$ ( $l$ being one of its components) the vector that contains all the continuous components. We consider a hybrid state vector $s_{k}$ where the subscript is used to indicate a sample:

$$
s_{k}=\left[\begin{array}{ll}
\mathcal{I}_{k} & x_{k}
\end{array}\right]^{T} \in \mathbb{N} \times \mathbb{R}^{n}
$$

As done in [11], such an observation problem can be described by a stochastic model:

$$
\begin{cases}\mathcal{I}_{k+1} & \sim P\left(\mathcal{I}_{k+1}=\mathcal{I} \mid \mathcal{I}_{k}, x_{k}, y_{k}\right) \\ x_{k+1} & =f\left(\mathcal{I}_{k}, x_{k}\right)+\alpha_{k} \\ y_{k} & =g\left(\mathcal{I}_{k}, x_{k}\right)+\beta_{k}\end{cases}
$$

Where $y_{k}$ is the observation at step $k$ using the sensors, $\alpha_{k}$ the process error and $\beta_{k}$ the observation error. $f($.$) is$ the process model describing the evolution of the continuous components and $g($.$) the observation model. These models$ depend on the carriageway ID because of the map constraint. Finally, $P($.$) is a transition kernel depicting the evolution of$ the probability of the IDs $\mathcal{I}$ contained in the map. In the following, a first order Markov chain assumption depending on the network topology is done. Thus, (2) is a Jump Markov System.

\section{B. Sequential estimation}

The problem is to estimate $p\left(s_{k} \mid y_{1: k}\right)$ sequentially using all the available measurements $y_{1: k}$ [12]. Considering the Bayes' factorization, the posterior density can be rewritten as:

$$
p\left(s_{k} \mid y_{1: k}\right)=p\left(y_{k} \mid s_{k}\right) \cdot \frac{p\left(s_{k} \mid y_{1: k-1}\right)}{p\left(y_{k} \mid y_{1: k}\right)}
$$

Where $p\left(y_{k} \mid s_{k}\right)$ is the observation likelihood according to the observation model of (2) and $p\left(y_{k} \mid y_{1: k}\right)$ a normalization factor given by:

$$
p\left(y_{k} \mid y_{1: k-1}\right)=\int p\left(y_{k} \mid s_{k}\right) \cdot p\left(s_{k} \mid y_{1: k-1}\right) \cdot d s_{k}
$$

Eq. (3) is the estimation step of the Bayesian recursive estimation process. This step needs $p\left(s_{k} \mid y_{1: k-1}\right)$, the prior density of $s_{k}$. Using the process model and the posterior at step $k-1$ :

$$
p\left(s_{k} \mid y_{1: k-1}\right)=\int p\left(s_{k} \mid s_{k-1}\right) p\left(s_{k-1} \mid y_{1: k-1}\right) d s_{k-1}
$$

We will see later that the prediction of continuous components depends on the previous state $s_{k-1}$, but also on the current carriageway. Eq. (3) and (5) constitute the solution of Bayesian MHMM.

\section{Estimation of the global positioning}

The global position can be estimated using the marginal density $p\left(x_{k} \mid y_{1: k}\right)$ of the continuous components:

$$
p\left(x_{k} \mid y_{1: k}\right)=\int p\left(x_{k}, \mathcal{I}_{k} \mid y_{1: k}\right) \cdot d \mathcal{I}_{k}
$$

The joint posterior $p\left(x_{k}, \mathcal{I}_{k} \mid y_{1: k}\right)$ can be factorized according to the Bayes' rule:

$$
p\left(x_{k}, \mathcal{I}_{k} \mid y_{1: k}\right)=p\left(x_{k} \mid \mathcal{I}_{k}, y_{1: k}\right) p\left(\mathcal{I}_{k} \mid y_{1: k}\right)
$$

This factorization isolates the problem of carriageway identification, described by $p\left(\mathcal{I}_{k} \mid y_{1: k}\right)$, from the problem of localizing the mobile on the carriageway, depicted by $p\left(x_{k} \mid \mathcal{I}_{k}, y_{1: k}\right)$. By considering that $p\left(\mathcal{I}_{k} \mid y_{1: k}\right)$ is a discrete density (with Dirac functions) and by introducing Eq. (7) in Eq. (6), one can easily find that the marginal density is a weighted sum over all the carriageways contained in the map:

$$
p\left(x_{k} \mid y_{1: k}\right)=\sum_{i=1}^{N_{\mathcal{I}}} p\left(x_{k} \mid \mathcal{I}_{k}=\mathcal{I}, y_{1: k}\right) P\left(\mathcal{I}_{k}=\mathcal{I} \mid y_{1: k}\right)
$$

Where $N_{\mathcal{I}}$ is the number of carriageways.

Eq. (8) gives the expression that allows finding to most likely solution on the whole map. This strategy is not always the best, especially if the identification of the road is ambiguous. In this case, it can be better to do an estimation par carriageway. For a given carriageway $\mathcal{I}$, the positioning hypothesis $M_{k}^{\mathcal{I}}$ is characterized by its posterior and its probability with respect to the others:

$$
M_{k}^{\mathcal{I}}=\left\{\begin{array}{l}
p\left(x_{k} \mid \mathcal{I}_{k}=\mathcal{I}, y_{1: k}\right) \\
\Omega_{k}^{\mathcal{I}}=P\left(\mathcal{I}_{k}=\mathcal{I} \mid y_{1: k}\right)
\end{array} \quad \text { with } \sum_{i=1}^{N_{\mathcal{I}}} \Omega_{k}^{\mathcal{I}}=1\right.
$$

\section{SOLVING THE MHMM USING RAW GPS AND POLYLINES}

In this section, we show how to solve the Bayesian MHMM when using raw GPS measurements and when the carriageways are describes by polylines. A measure of the speed of the vehicle is also used.

We use a sequential Monte Carlo method to solve the MHMM problem. To implement efficiently the tracking, a marginalized PF can be applied [11], [13], [14]. Indeed, considering the factorization of $p\left(s_{k} \mid y_{1: k}\right)$ given by Eq. (7), the estimation of the continuous state can be isolated from the discrete state estimation. So, hypotheses are attached to every candidate road and EKF is used to track the continuous components of the state vector. New candidate roads are randomly chosen at junctions using the connection information stored in the map. This is a sub-optimal approach, but it allows reducing strongly the number of particles. 


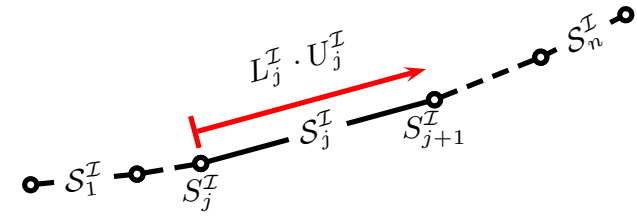

Fig. 1: Geometrical description of a carriageway.

\section{A. State description}

Since we use raw GPS measurements to compute the Bayesian inference, additional continuous parameters are needed, so :

$$
x_{k}=\left[\begin{array}{llll}
l_{k} & v_{k} & d_{k} & \dot{d}_{k}
\end{array}\right]
$$

where $v_{k}$ is the speed along the carriageway. $d_{k}$ and $\dot{d}_{k}$ represent the clock offset and clock drift of the receiver.

1) Evolution of the carriageway IDs: The kernel transition for the carriageway IDs $\mathcal{I}$ is given by a topological approach: if the hypothesis reaches the end of carriageway, the transition depends on the connected carriageways $\mathcal{C}^{\mathcal{I}}$. By assuming an equiprobable transition:

$$
\left\{\begin{array}{l}
P\left(\mathcal{I}_{k+1}=\mathcal{I}_{k} \mid \mathcal{I}_{k}, l_{k}<L^{\mathcal{I}_{k}}\right)=1 \\
P\left(\mathcal{I}_{k+1}=\mathcal{J} \mid \mathcal{I}_{k}, l_{k} \geq L^{\mathcal{I}_{k}}\right)=\frac{1}{\operatorname{dim} \mathcal{C}^{\mathcal{I}}} \quad \forall \mathcal{J} \in \mathcal{C}^{\mathcal{I}}
\end{array}\right.
$$

Where $L^{\mathcal{I}_{k}}$ is the total length of the carriageway $\mathcal{I}_{k}$.

2) Evolution of continuous components: As constrained hypotheses are used, the process model $x_{k+1}=f\left(\mathcal{I}_{k}, x_{k}\right)$ is reduced to a conditionally linear model. The nonlinearity is due to switching.

$$
x_{k+1}= \begin{cases}A \cdot x_{k}+B \cdot L^{\mathcal{I}_{k}} & \text { if } \mathcal{I}_{k+1} \neq \mathcal{I}_{k} \\ A \cdot x_{k} & \text { else }\end{cases}
$$

The process matrices $A$ and $B$ are invariant and given by

$$
A=\left[\begin{array}{cccc}
1 & T_{e} & 0 & 0 \\
0 & 1 & 0 & 0 \\
0 & 0 & 1 & T_{e} \\
0 & 0 & 0 & 1
\end{array}\right] \text { and } B=\left[\begin{array}{c}
-1 \\
0 \\
0 \\
0
\end{array}\right]
$$

$T_{e}$ being the sampling period.

\section{B. Constrained observation models}

The main idea here is to use the raw GPS measurements, PR and Dopplers, and the speed of the vehicle to estimate the likelihood of every hypothesis. The map acts as a constraint. Let consider a polyline composed by $n+1$ segments: each segment is defined by its origin $S_{i}^{\mathcal{I}}$, its length $L_{i}^{\mathcal{I}}$ and its orientation in space $U_{i}^{\mathcal{I}}$ (see Figure (1)). The last segment is set with $L_{n+1}^{\mathcal{I}}=0$. To find the global position $X_{k}^{\mathcal{I}}$ we first need to extract the segment indexed $j$ such that :

$$
\sum_{i=1}^{j} L_{i}^{\mathcal{I}}<l<\sum_{i=1}^{j+1} L_{i}^{\mathcal{I}}
$$

The constrained PR model for one satellite is obtained by using the carriageway geometry in the classical PR model [15]:

$$
\rho_{k}^{\mathcal{I}}=\left\|S_{j}^{\mathcal{I}}+\left(l_{k}-\sum_{i=1}^{j-1} L_{i}^{\mathcal{I}}\right) U_{j}^{\mathcal{I}}-X_{k}^{s}\right\|+d_{k}
$$

Where $X_{k}^{s}$ is the satellite position reconstructed from the broadcast ephemeris.

The same approach is used for the Doppler measurements. We assume here that velocity is also constrained: the vehicle direction is assumed to be collinear to shape segment direction. The Doppler measurement is given by a dot product:

$$
\dot{\rho}_{k}^{\mathcal{I}}=\left(v_{k} U_{k}^{\mathcal{I}}-V_{k}^{s}\right) \bullet u_{\mathrm{los}}+\dot{d}_{k}
$$

Where $V_{k}^{s}$ is the satellite velocity obtained from the broadcast ephemeris and $u_{\mathrm{los}}$ is the line-of-sight vector. $u_{\text {los }}$ depends also on the map geometry since the position is constraint:

$$
u_{\operatorname{los}}=\frac{S_{j}^{\mathcal{I}}+\left(l_{k}-\sum_{i=1}^{j-1} L_{i}^{\mathcal{I}}\right) U_{j}^{\mathcal{I}}-X_{k}^{s}}{\left\|S_{j}^{\mathcal{I}}+\left(l_{k}-\sum_{i=1}^{j-1} L_{i}^{\mathcal{I}}\right) U_{j}^{\mathcal{I}}-X_{k}^{s}\right\|}
$$

Additionally, the vehicle speed obtained from CAN bus is used:

$$
y_{C A N, k}=\left[\begin{array}{llll}
0 & 1 & 0 & 0
\end{array}\right] \cdot x_{k}
$$

Next, speed and GPS errors are assumed to be independent.

\section{Marginalized particle filter}

1) Continuous Components Tracking: As EKF estimates the continuous components, errors are assumed to be Gaussian. Thus, process and observation errors are modeled by Gaussian white noises: $\alpha_{k} \sim \mathcal{N}\left(0, Q_{\alpha}\right)$ and $\beta_{k} \sim$ $\mathcal{N}\left(0, Q_{\beta}\right)$. The candidate positions are approximated by Gaussian densities along the carriageways. Their mean $\mu_{k \mid k}$ and covariance $\Sigma_{k \mid k}$ define the density:

$$
p\left(x_{k \mid k} \mid \mathcal{I}_{k}, y_{1: k}\right) \simeq \mathcal{N}\left(\mu_{k \mid k}, \Sigma_{k \mid k} \mid \mathcal{I}_{k}\right)
$$

$\mu_{k \mid k}$ and $\Sigma_{k \mid k}$ are given by [12]:

$$
\begin{cases}K & =\Sigma_{k \mid k-1} \cdot G^{t} \cdot\left(G \cdot \Sigma_{k \mid k-1} \cdot G^{t}+Q_{\beta}\right)^{-1} \\ \mu_{k \mid k} & =\mu_{k \mid k-1}+K\left(y_{k}-g\left(\mathcal{I}_{k}, \mu_{k \mid k-1}\right)\right) \\ \Sigma_{k \mid k} & =(\mathrm{I}-K \cdot G) \cdot \Sigma_{k \mid k-1}\end{cases}
$$

Where $G=\frac{\partial g}{\partial x}$ is the Jacobian matrix of the observation model.

The prediction stage is given by:

$$
\begin{cases}\mu_{k \mid k-1} & =f\left(\mathcal{I}_{k}, \mu_{k-1 \mid k-1}\right) \\ \Sigma_{k \mid k-1} & =A \cdot \Sigma_{k-1 \mid k-1} \cdot A^{t}+Q_{\alpha}\end{cases}
$$




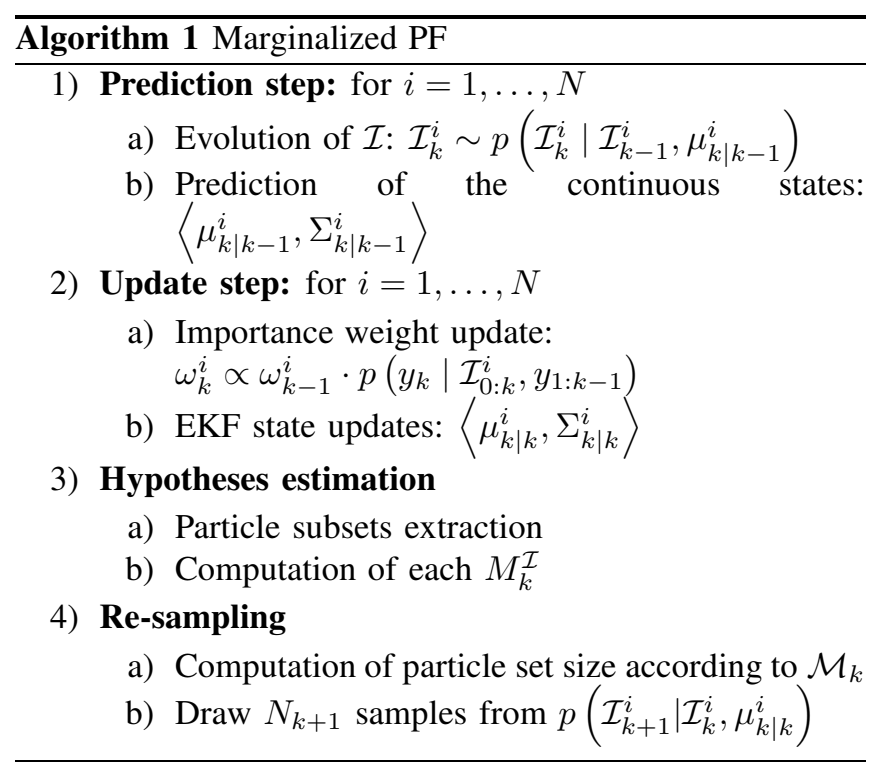

2) Estimation of the IDs: For this discrete problem, we do importance sampling. Let consider a set of $N$ weighted samples of $p\left(\mathcal{I}_{k} \mid y_{1: k}\right)$ :

$$
\chi_{k}=\left\{\mathcal{I}_{k}^{i}, \omega_{k}^{i}\right\}_{i=1: N}
$$

Where $\omega_{k}^{i}$ denotes the weight. Therefore, the density describing the road identification problem is given by:

$$
p\left(\mathcal{I}_{k} \mid y_{1: k}\right)=\sum_{i=1}^{N} \omega_{k}^{i} \cdot \delta_{r_{k}}\left(\mathcal{I}_{k}^{i}\right)
$$

where $\delta_{r_{k}}$ is the Dirac function.

To estimate the weight of each particle, standard bootstrap [16] is applied for the recursion:

$$
\omega_{k}^{i} \propto \omega_{k-1}^{i} \cdot p\left(y_{k} \mid \mathcal{I}_{0: k}^{i}, y_{1: k-1}\right)
$$

The measurement likelihood $p\left(y_{k} \mid \mathcal{I}_{0: k}^{i}, y_{1: k-1}\right)$ is quantified using two Normal laws:

$$
\begin{gathered}
p\left(y_{k} \mid \mathcal{I}_{0: k}^{i}, y_{1: k-1}\right) \simeq \\
\mathcal{N}\left(y_{C A N, k}, Q_{C A N, k} \mid \mathcal{I}_{k}^{i}\right) \cdot \mathcal{N}\left(y_{G P S, k}, Q_{G P S, k} \mid \mathcal{I}_{k}^{i}\right)
\end{gathered}
$$

The likelihood is computed according to the predicted measurement of the EKF [14].

3) Algorithm: Algorithm 1 describes the realization of the marginalized PF. A positioning hypothesis (9) is obtained from the subset $\chi_{k}^{\mathcal{I}}$ of the particles evolving on the given carriageway $\mathcal{I}$ through a Gaussian mixture:

$$
M_{k}^{\mathcal{I}}=\left\{\begin{array}{l}
\sum_{i=1}^{N_{k}^{\mathcal{I}}} \omega_{k}^{i} \cdot \mathcal{N}\left(\mu_{k \mid k}^{i}, \Sigma_{k \mid k}^{i} \mid \mathcal{I}_{k}^{i}=\mathcal{I}\right) \\
\Omega_{k}^{\mathcal{I}}=\sum_{i=1}^{N_{k}^{\mathcal{I}}} \omega_{k}^{i}
\end{array}\right.
$$

Where $N_{k}^{\mathcal{I}}$ is the dimension of this particle subset.

To avoid particle set degeneracy, a re-sampling strategy is used. We have chosen here the adaptive approach proposed by the authors of [17]. The size of the particle set is

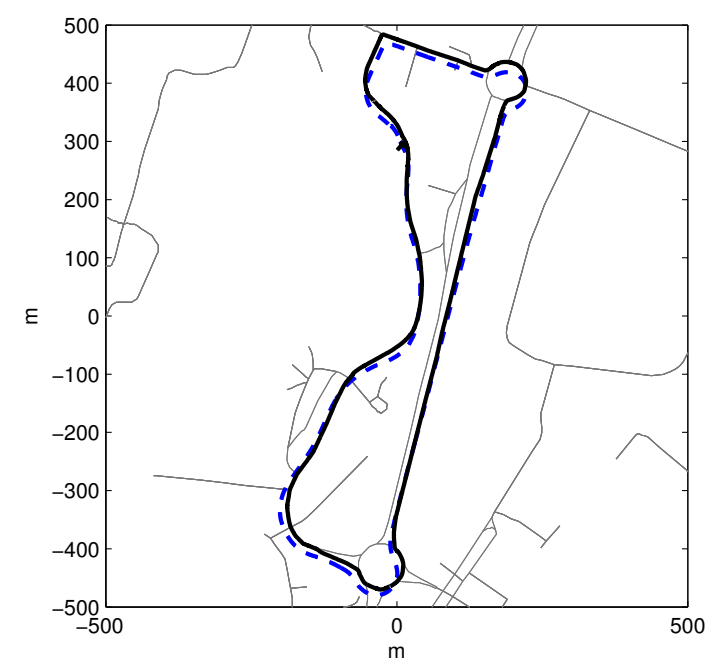

Fig. 2: Trajectory -dashed-vs. map-matched trajectory plain - in a ENU frame centered on the map.

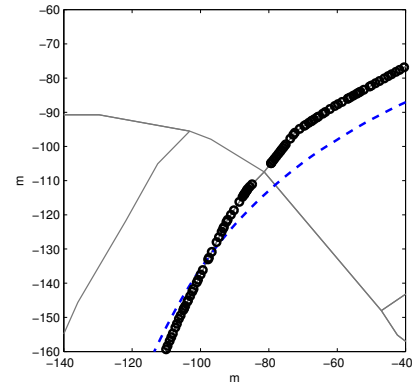

(a) With Dopplers

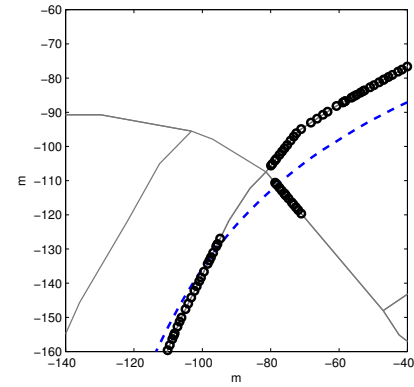

(b) Without Dopplers
Fig. 3: Road junction crossing: vehicle path -dashed-vs. map-based position of the most-likely hypothesis $-d o t$-.

then adjusted according to the size of the hypotheses set. When approaching a junction for instance, $\mathrm{N}$ is automatically increased.

\section{EXPERIMENTAL RESULTS}

In this section, several experimental results are reported to show the performance of the MHMM based on raw GPS measurements and a measure of the speed of the vehicle. Tests have been carried out in Compiègne, with an experimental car. The raw data of a GPS PolaRx $2 e$ receiver has been logged simultaneously with the speed of the vehicle at $10 \mathrm{~Hz}$. The data has been then post-processed in a EastNorth-Up (ENU) frame centered on the map.

In a first test, a crossroads is studied to illustrate the behavior of the method. Then, the full trial is evaluated. Finally, the performance with a degraded satellite visibility is assessed. The results of the road selection are compared to a hand-made map-matching similar to the one presented in [10] and estimated continuous state are compared with the positioning output of the PolaRx $2 e$. Figure 2 shows the true trajectory -dashed-versus the map-matched trajectory -bold. The TeleAtlas map used here presents roughly an offset of 15 meters. 


\begin{tabular}{|c|c|c||c|c||c|c|c|}
\hline & \multicolumn{2}{|c|}{$\operatorname{dim} \mathcal{M}_{k}=1$} & \multicolumn{2}{c||}{$\operatorname{dim} \mathcal{M}_{k} \geq 2$} & \multicolumn{3}{c|}{ Total } \\
& OK & NOK & Amb. & NOK & OK & Amb. & NOK \\
\hline Mean & $97 \%$ & $3 \%$ & $98 \%$ & $2 \%$ & $89 \%$ & $8 \%$ & $3 \%$ \\
Best & $99 \%$ & $1 \%$ & $100 \%$ & $0 \%$ & $90 \%$ & $9 \%$ & $1 \%$ \\
Worst & $93 \%$ & $7 \%$ & $96 \%$ & $4 \%$ & $87 \%$ & $7 \%$ & $6 \%$ \\
\hline
\end{tabular}

TABLE I: Road Identification
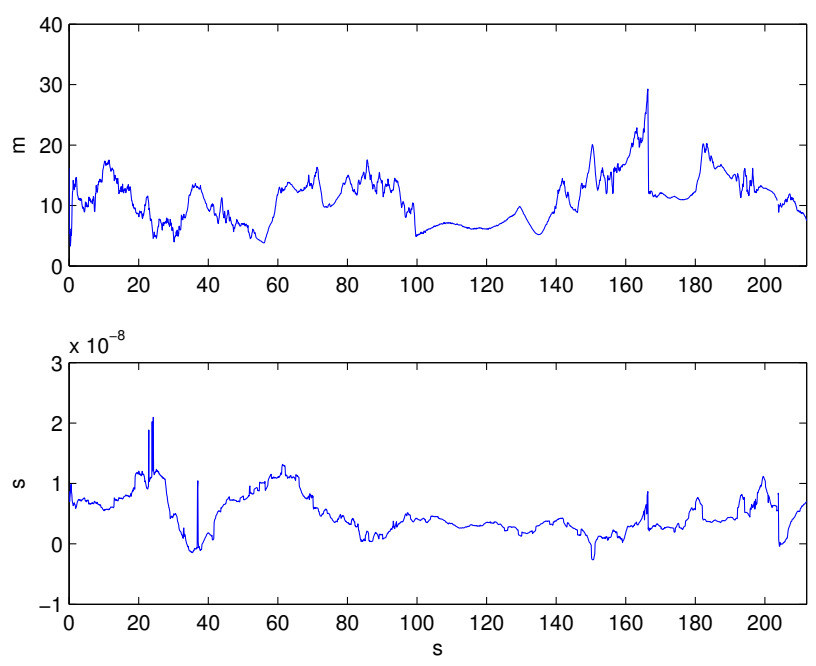

Fig. 4: Estimation results for first order parameters with a good visibility: Position $-u p-$ and clock offset - down-.
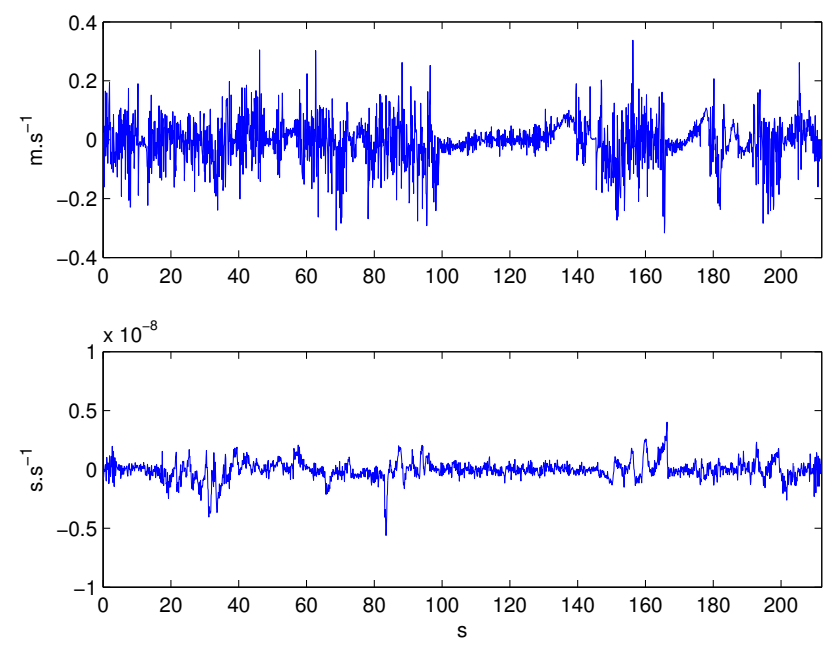

Fig. 5: Estimation results for derivative parameters with a good visibility: Velocity $-u p-$ and clock drift - down-.

\section{A. Behavior at a road junction}

Figure 3 shows an intersection crossing. Two cases are considered: Algorithm 1 using Doppler measurements $-3 a-$ and without Dopplers $-3 \mathrm{~b}-$. Without Dopplers, the road identification only relies on the particles positions through the constrained PR model (15). Thus, several wrong selections are done at the junction as the map suffers from biases. At the same junction, the road identification is correctly done when relying on position and heading information provided by the Dopplers (16). This shows clearly the benefits of using Dopplers to enhance the map-matching process: The particle score is also estimated according to the heading error, giving additional parameters to discard wrong hypotheses.

\section{B. Performance under normal conditions}

1) Road identification: The performance of the proposed method is here evaluated with a good satellite visibility. As a the solver acts like a Monte Carlo method, the data-set was processed hundred times to obtain a statistical description of the road identification performance. Due to the limited accuracy of the road selection ground truth, results were rounded to the nearest percent. Table I shows these results:

- OK: Road identification is correctly done: the correct road is the only hypothesis.

- Amb.: An ambiguous area is identified and the hypotheses set contains the correct road.

- NOK: Wrong selection: the correct ID doesn't appear in the hypotheses set.

In spite of the map offset, the method shows interesting performance as the wrong selection rate stands low. Moreover, ambiguous areas are well identified, and the best and worst cases have similar rates. These results shows that the proposed method allows a correct identification of ambiguous areas and an efficient road identification despite the low map accuracy.

2) Positioning quality: Road identification is only one aspect of the problem. Performance of the continuous state tracking has also to be evaluated: We consider here the mean state of the particle set. Figure 4 shows the estimation errors for the first order parameters: position and receiver clock offset. Additionally, Figure 5 provides the estimation errors for the derivative parameters: velocity and clock drift.

Considering the first order parameters, the estimation errors are not centered because of the tight integration of the map in the fusion process. The mean value for the position is between $12 \mathrm{~m}$ and $15 \mathrm{~m}$. This bias is due to the map offset. Identically, the clock offset estimation error is not centered, meaning that part of the map offset is reported to the clock offset estimation. Conversely, estimation errors on the derivative parameters are centered, meaning that the map constraint as a limited impact on these parameters. These results show that the proposed method performs efficiently the continuous state tracking under open-sky.

\section{Performance with few visible satellites}

We have removed here satellites from the computation to simulate urban canyons.

1) 3 satellites available: Table II shows the results of the road identification behavior and figure 6 the positioning error in the best case. Considering the road identification, no significant variation is observed except for the worst case that shows a significant increase of wrong selections. The reduction of the redundancy in the measurement domain can explain this degradation: as the measurement redundancy is reduced, discarding wrong hypothesis takes more time.

By considering the positioning error in the best case, one can notice that the positioning quality is similar to open-sky conditions. Regarding this result, one can consider that with only 3 satellites providing Dopplers and PR, the system is able to track efficiently the rad ID and the vehicle position. 


\begin{tabular}{|c|c|c||c|c||c|c|c|}
\hline & \multicolumn{2}{|c||}{$\operatorname{dim} \mathcal{M}_{k}=1$} & \multicolumn{2}{c||}{$\operatorname{dim} \mathcal{M}_{k} \geq 2$} & \multicolumn{3}{c|}{ Total } \\
& OK & NOK & Amb. & NOK & OK & Amb. & NOK \\
\hline Mean & $95 \%$ & $5 \%$ & $97 \%$ & $3 \%$ & $84 \%$ & $12 \%$ & $4 \%$ \\
Best & $99 \%$ & $1 \%$ & $99 \%$ & $1 \%$ & $87 \%$ & $12 \%$ & $1 \%$ \\
Worst & $88 \%$ & $12 \%$ & $94 \%$ & $8 \%$ & $77 \%$ & $12 \%$ & $11 \%$ \\
\hline
\end{tabular}

TABLE II: Road identification with 3 satellites.

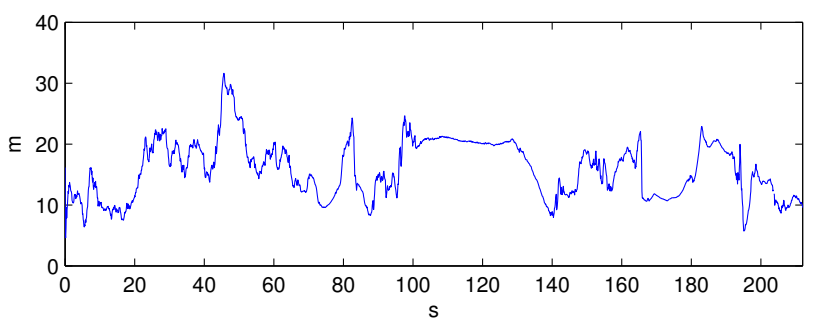

Fig. 6: Positionning error with 3 satellites in the best case.

2) 2 satellites available: With only 2 satellites, the identification performance significantly decreases as shown by Table III. Moreover, the dispersion of the results is significantly increased: the worst case shows about $50 \%$ of wrong selection whereas the best case shows similar results than previously. Theses results come from the lack of redundancy with only 2 satellites. In such a case, only 5 measurements are used to estimate the state vector, the dimension of which is 5. Therefore, without redundancy, it is possible to find an erroneous hypothesis that is more likely than the "true" one.

Figure 7 describes the positioning errors in the best case. Errors are similar to those observed for the mean case with only three satellites. This is an interesting result: when road identification is correctly solved, the method is able to track the vehicle states with only two measurements. Thus, we conclude that information redundancy is mandatory to solve road identification, but less data is needed for position tracking.

\section{CONCLUSiON}

In this paper, a generic approach for MHMM has been presented. Based on a tight integration of a navigable road map with sensor measurements, this approach allows multihypothesis tracking of map-matched solutions in a Bayesian framework. Here, topological description is used to predict hypotheses and geometrical description is used to express constrained observation models. Such an approach allows solving road identification and positioning simultaneously.

This method has been applied to the specific problem of directly map-matching GPS measurements using a marginalized PF that is able to track several hypotheses. Here, a 3D standard road map has been used to constraint the location space. Experimental results show interesting performance even under reduced visibility conditions since this approach is able to provide a positioning even if only two satellites are visible. Moreover, benefits of Dopplers measurements have been highlighted. As they provide additional redundancy through heading information, they enhance wrong hypotheses elimination. Finally, the results show that such an approach is highly dependent on the map quality: the

\begin{tabular}{|c|c|c||c|c||c|c|c|}
\hline & \multicolumn{2}{|c||}{$\operatorname{dim} \mathcal{M}_{k}=1$} & \multicolumn{2}{c|}{$\operatorname{dim} \mathcal{M}_{k} \geq 2$} & \multicolumn{3}{c|}{ Total } \\
& OK & NOK & Amb. & NOK & OK & Amb. & NOK \\
\hline Mean & $83 \%$ & $17 \%$ & $86 \%$ & $14 \%$ & $71 \%$ & $13 \%$ & $16 \%$ \\
Best & $97 \%$ & $3 \%$ & $95 \%$ & $5 \%$ & $84 \%$ & $12 \%$ & $4 \%$ \\
Worst & $53 \%$ & $47 \%$ & $43 \%$ & $57 \%$ & $44 \%$ & $7 \%$ & $49 \%$ \\
\hline
\end{tabular}

TABLE III: Road identification with 2 satellites.

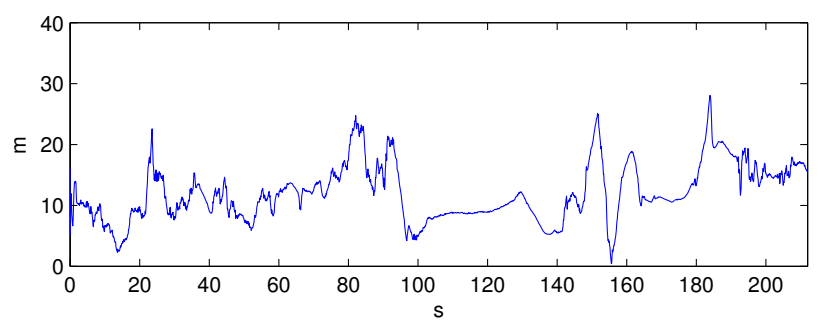

Fig. 7: Positionning error with 2 satellites in the best case.

use of enhanced maps should greatly improve positioning performance. Further development will consider the integrity monitoring of the positioning hypotheses as this information is of great interest for many ITS applications.

\section{REFERENCES}

[1] M. Quddus, W. Ochieng, and R. Noland, "Current map-matching algorithms for transport applications: State-of-the art and future research directions," Transportation Research Part C: Emerging Technologies, vol. 15, no. 5, pp. 312-328, 2007.

[2] C. Smaili, M. El Najjar, and F. Charpillet, "A road matching method for precise vehicle localization using hybrid bayesian network," Journal of Intelligent Transportation Systems, vol. 12, no. 4, October 2008.

[3] J. S. Greenfeld, "Matching gps observation to locations on a digital map," Transp. Res. Board, 81st Annual Meeting of the, 2002.

[4] R. Toledo-Moreo, D. Bétaille, and F. Peyret, "Lane-level integrity provision for navigation and map matching with GNSS, dead reckoning and enhanced maps," Intelligent Transportation Systems, IEEE Transactions on, vol. 11, no. 1, pp. 100-112, March 2010.

[5] S. Syed, "Gps-based map matching in the pseudorange measurement domain," in Proc. of GNSS 2004, Long Beach, CA, 2004.

[6] C. Fouque and P. Bonnifait, "Road navigation system monitoring using a pseudorange snapshot test," in IFAC World Congress 2008, vol. 17, no. 1, COEX, South Korea, 2008.

[7] Y. Cui and S. Ge, "Autonomous vehicle positioning with gps in urban canyon environments," IEEE TRO, vol. 19, pp. 15-25, 2003.

[8] G. Taylor and G. Blewitt, Intelligent Positioning: GIS-GPS Unification. London, UK: John Wiley and Sons Ltd., 2006.

[9] Maged Jabbour, Philippe Bonnifait, and Véronique Cherfaoui, "MapMatching Integrity using Multi-Sensor Fusion and Multi-Hypothesis Road Tracking," J Intell Transport Syst Tech Plann Oper, vol. 12, no. 4, pp. 189-201, 102008.

[10] P. Bonnifait, J. Laneurit, and C. Fouque, "Multi-hypothesis mapmatching using particle filtering," in ITS World Congress, Stockholm, Sept. 2009.

[11] N. de Freitas, R. Dearden, F. Hutter, R. Morales-Menendez, J. Mutch, and D. Poole, "Diagnosis by a waiter and a mars explorer," in Proceedings of the IEEE, special issue on Sequential State Estimation, vol. 92, no. 3, 2003, pp. 455 - 468.

[12] S. Thrun, W. Burgard, and D. Fox, Probabilistic Robotics. The MIT Press, 2005.

[13] C. Andrieu, M. Davy, and A. Doucet, "Efficient particle filtering for jump markov systems - applications to time-varying autoregressions," IEEE Trans. Signal Processing, vol. 51, no. 7, pp. 1762-1770, 2003.

[14] T. Schön, F. Gustafsson, and P.-J. Nordlund, "Marginalized Particle Filters for Mixed Linear Nonlinear State-Space Models," IEEE Trans. on Signal Processing, vol. 53, pp. 2279-2289, 2005.

[15] N. Samama, Global Positionning: Technologies and Performance. Wiley-Interscience, 2008.

[16] B. Ristic, S. Arulampalam, and N. Gordon, Beyond the Kalman Filter. Particle Filters for Tracking Applications. Artech House, 2004.

[17] D. Fox, "Kld-sampling: Adaptive particle filters," in In NIPS, M. Press, Ed., vol. 14, 2001, pp. 713-720. 